THURSDAY, 15 JUNE 2017 Fifty shades of remission in RA

\section{OP0192 ADDING ULTRASOUND TO THE TREAT-TO-TARGET STRATEGY SHOWS NO BENEFIT IN ACHIEVEMENT OF REMISSION: RESULTS FROM THE BIODAM COHORT}

A. Sepriano ${ }^{1}$, S. Ramiro ${ }^{1}$, R. Landewé ${ }^{2}$, D. van der Heijde ${ }^{1}$, S. Ohrndorf ${ }^{3}$, O. FitzGerald ${ }^{4}$, M. Backhaus ${ }^{3}$, M. Larché ${ }^{5}$, J. Homik ${ }^{6}$, A. Saraux ${ }^{7}$, H. Hammer ${ }^{8}$, L. Terslev $^{9}$, M. Østergaard ${ }^{10}$, G. Burmester ${ }^{3}$, B. Combe ${ }^{11}$, M. Dougados ${ }^{12}$, C. Hitchon ${ }^{13}$, G. Boire $^{14}$, R. Dadashova ${ }^{15}$, J. Paschke $^{15}$ E. Hutchings ${ }^{15}$, W. Maksymowych $6 .{ }^{1}$ LUMC, Leiden; ${ }^{2}$ ARC, Amsterdam, Netherlands: ${ }^{3}$ Charité, Berlin, Germany; ${ }^{4}$ SVUH, Dublin, Ireland; ${ }^{5}$ McMaster University, Hamilton; ${ }^{6}$ University of Alberta, Edmonton, Canada; ${ }^{7} \mathrm{CHU}$, Brest, France; ${ }^{8}$ Diakonhjemmet Hospital, Oslo, Norway; ${ }^{9}$ Copenhagen UH, Copenhagen; ${ }^{10}$ Copenhagen UH, Copenhagen, Denmark; ${ }^{11} \mathrm{CHU}$, Montpellier; ${ }^{12}$ Descartes University, Paris, France; ${ }^{13}$ University of Manitoba, Winnipeg; ${ }^{14}$ University of Sherbrooke, Quebec; ${ }^{15}$ CaRE Arthritis Ltd, Edmonton, Canada

Background: While, a Treat-to-Target strategy (T2T), treating patients with rheumatoid arthritis (RA) towards a certain target (eg. clinical remission; T2TREM), is highly recommended, several patients in clinical remission often have residual synovitis on ultrasound-imaging (US). This may result in silent radiographic progression and clinical flare. It is arguable whether targeting US-synovitis may result in "deeper" remission in clinical practice.

Objectives: To assess whether using US in a T2T strategy leads to more patients meeting clinical remission than using only clinical information.

Methods: Patients with RA who started or changed csDMARD and/or anti-TNF treatment followed in centers with expertise in US and participating in BIODAM (2-year multicenter prospective observational cohort) were included. Clinical and US data [by the US7-score that includes 7 joints of the clinically dominant hand and foot for synovitis and tenosynovitis on gray-scale US (GSUS) and power-doppler US (PDUS) and erosions on GSUS] were collected every 3 months. Per visit was decided whether the patient was treated according to the clinical definition of T2T with remission as benchmark (T2T-CLIN-REM). Though not mandatory, US-data could also be used for this purpose. T2T-CLIN-REM was considered correctly applied if: either i) a patient already had a disease activity score below the remission target (i.e. ACR/EULAR boolean remission) or ii) if not, treatment was intensified. A T2T strategy taking also US data into account (T2T-CLIN-US-REM) was considered correctly applied if: either i) both clinical and US remission (all joints included in the US7-score with GSUS synovitis $<2$ and PDUS synovitis=0) were present; or ii) if not, the treatment was intensified. The main outcome was ACR/EULAR boolean remission. The effect of adding US to T2T (T2T-CLIN-US-REM) on clinical remission 3 months later compared to a clinical remission benchmark only (T2T-CLIN-REM) was analyzed using generalized estimating equations with auto-regression.

Results: In total 963 visits of 130 patients were included. T2T-CLIN-US-REM was correctly followed in $33 \%$ of the visits, T2T-CLIN-REM in $14 \%$, and any of these in $52 \%$. Remission according to the ACR/EULAR-boolean definition was achieved in $19.6 \%$ of the visits. Compared to the conventional T2T-CLIN-REM strategy, using a combined clinical and US benchmark for T2T led to a lower - instead of higher - likelihood of ACR/EULAR-boolean remission 3 months later [OR (95\% Cl): 0.39 $(0.24 ; 0.63]$ (table).

Table. Effect of following a T2T strategy taking both clinical and US data into account (T2T-CLIN-US-REM) compared to the clinical definition of T2T (T2T-CLIN-REM, reference) in meeting the targets 3 months later and over a period of 2 years.

\begin{tabular}{|c|c|}
\hline T2T approach Outcome & $\begin{array}{c}\text { ACR/EULAR boolean remission } \\
\text { aOR* }(95 \% \mathrm{CI})\end{array}$ \\
\hline No T2T vs T2T-CLIN-REM alone ${ }^{6}$ & $0.49(0.27 ; 0.88)$ \\
\hline
\end{tabular}

T2T-CLIN-US-REM vs T2T-CLIN-REM alone

$0.39(0.24 ; 0.63)$

*Adjusted for age, gender, HAQ-score, RF, ACPA, previous use of DMARDs, smokingstatus, education and number of comorbidities. $§$ Clinical definition of T2T (T2T-CLINREM) was used as the comparator for each of the presented treatment strategies.

Conclusions: Our results, from a non-randomized study, did not suggest an advantage of using US of 7 joints in addition to clinical examination as a T2T benchmark as compared to clinical examination alone in getting RA patients into clinical remission.

Disclosure of Interest: None declared

DOI: 10.1136/annrheumdis-2017-eular.1613
THURSDAY, 15 JUNE 2017 Innate immunity OP0193 INFLAMMATION OF ADVENTITIAL NERVES OCCURS IN GIANT
CELL ARTERITIS PATIENTS AND IT IS CHARACTERIZED BY INFLAMMASOMES, UPR AND AUTOPHAGY ACTIVATION

F. Ciccia ${ }^{1}$, A. Rizzo ${ }^{1}$, R. Alessandro ${ }^{1}$, S. Raimondo ${ }^{1}$, G. Guggino ${ }^{1}$,

A. Cavazza ${ }^{2}$, C. Salvarani ${ }^{2}$, G. Triolo ${ }^{1} .{ }^{1}$ University of Palermo, Palermo;

${ }^{2}$ Arcispedale Santa Maria Nuova, Reggio Emilia, Italy

Background: Vascular adventitia is a major site of immune surveillance and inflammatory cell trafficking and is the most complex compartment of the vessel wall comprising fibroblasts, dendritic cells and macrophages, progenitor cells, vasa vasorum, pericytes and adrenergic nerves. It has been proposed that activation of adventitial nerves and release of sensory neuropeptides from their peripheral terminals may leads to neurogenic inflammation. Giant cell arteritis (GCA) is an immune-mediated disease of unknown etiology in which the inflammatory process seems to start from the adventitia of affected arteries.

Objectives: aim of the study was to evaluate the occurrence of adventitial nerves inflammation and to immunologically characterize the adventitial neuritis occurring in GCA patients.

Methods: Immunohistochemistry and RT-PCR were used to study the presence of neuritis in temporal artery samples obtained from 30 patients with temporal artery (TAB) positive GCA, 20 TAB negative GCA and 20 controls. Laser capture microdissection (LCM) was used to isolate adventitial nerves and nerve expression of pro-inflammatory cytokines involved in the pathogenesis of GCA such as IL-6, IL-17, IL-32, IL-9, IL-33 was assessed by RT-PCR. Expression of pro-inflammatory cytokines by adventitial nerves was further studied by immunohistochemistry and confocal microscopy. Autophagy, unfoled protein response (UPR) and inflammasomes pathways were also studied by RT-PCR and immunohistochemistry.

Results: Adventitial nerves showed infiltration of $\mathrm{CD}^{+} \mathrm{T}$ cells in all the TAB positive and in 12 out of 20 TAB negative arteries but were never observed in control arteries. RT-PCR expression analysis of different proinflammatory cytokines clearly demonstrated specific over-expression of IL-33 in LCM isolated inflamed nerves. Immunohistochemical and confocal microscopy analysis confirmed nerve IL-33 expression. RT-PCR and immunoistochemistry demonstrated that AIM2 and NLRP3, but not NLCR4, inflammasomes were activated in inflamed nerves of GCA patients. According to inflammasomes activation, increased IL-18 expression was observed. Autophagy-related genes such as ATG16L1 and LC3 and UPR-related genes such as XBP-1 and CHOP were also over-expressed in GCA adventitial nerves as demonstrated by RT-PCR and immunohistochemistry. Finally, increased expression of AIM2 and NLRP3 inflammasomes, autophagy and UPR was also observed outside the nerves, in the context of inflamed artery wall.

Conclusions: here we demonstrated that adventitial neuritis is present in both inflamed and non-inflamed arteries of GCA patients. GCA inflamed nerves specifically produce IL-33 a cytokine of the innate immunity that has been demonstrated to be involved in the pathogenesis of GCA. AIM2 and NLRP3 inflammasomes activation was also observed in GCA arteries and in particular in the inflamed adventitial nerves being accompanied by the increased expression of IL-18. Specific activation of autophagy and UPR pathways was also observed in the inflamed GCA nerves. Altogheter these findings seem to suggest a complex immune activation of adventitial nerves in GCA, suggesting a possible role of arterial neuritis in the initiation and perpetuation of GCA artery inflammation.

Disclosure of Interest: None declared

DOI: 10.1136/annrheumdis-2017-eular.4345

\section{OP0194 INVOLVEMENT OF TOLL-LIKE RECEPTOR 9 IN THE PATHOGENESIS OF EROSIVE AUTOIMMUNE ARTHRITIS AND DURING OSTEOCLASTOGENESIS}

A. Fischer, B. Meyer, B. Niederreiter, G. Steiner. Internal Medicine III-Rheumatology, Medical University Vienna, Vienna, Austria

Background: Release and insufficient removal of endogenous nucleic acids may be involved in triggering harmful autoimmune reactions important in the initiation of systemic autoimmune diseases including rheumatoid arthritis (RA). Nucleic acid sensing molecules, such as the endosomal Toll-like receptors (TLRs) 7 and 9 , have been linked to pathogenic autoimmune processes, particularly in systemic lupus erythematosus, but their role in RA is less clear. Results previously obtained in rats with pristane-induced arthrits (PIA) suggested involvement of TLR9 in the pathogenesis of this arthritis model (1). Interestingly, rats with PIA develop autoantibodies associated with RA including rheumatoid factor, anti-RA33 and antibodies to carbamylated proteins (2).

Objectives: To gain more insight into the role of TLR9 in the pathogenesis of autoimmune arthritis by investigating the effects of TLR9 inhibition in rats with PIA.

Methods: Arthritis was induced in DA rats with the mineral oil pristane. Rats were treated with a TLR9 antagonist every other day, starting one before disease induction. Arthritis was scored using established scoring systems, inflammation and bone erosion were quantified by histological analysis. Expression of TLR9 